\begin{tabular}{|c|c|}
\hline Title & Memory and naive B-cell subsets in patients with multiple sclerosis \\
\hline Author(s) & Niino, Masaaki; Hirotani, Makoto; Miy azaki, Y usei; Sasaki, Hidenao \\
\hline Citation & $\begin{array}{l}\text { Neuroscience Letters, } 464 \text { (1), } 74.78 \\
\text { https://doi.org/10.1016/. .neulet.2009.08.010 }\end{array}$ \\
\hline Issue Date & 2009-10-16 \\
\hline Doc URL & http:/hdl.handle.net/2115/39404 \\
\hline Type & article (author version) \\
\hline File Information & NL4641_p74-78.pdf \\
\hline
\end{tabular}

Instructions for use 


\section{Memory and naïve B-cell subsets in patients with multiple sclerosis}

Masaaki Niino*, Makoto Hirotani, Yusei Miyazaki, Hidenao Sasaki

Department of Neurology, Hokkaido University Graduate School of Medicine, Sapporo, Japan

Number of text pages (including figures and tables): 13 pages

Number of figures and tables: 5 figures and 0 tables

* Corresponding author. Department of Neurology, Hokkaido University Graduate School of Medicine, Kita 15, Nishi 7, Kita-ku Sapporo, Japan.

Tel.: +81-11-706-6028; fax.: +81-11-700-5356

E-mail address: niino@med.hokudai.ac.jp (M. Niino)

Acknowledgement: This work was supported in part by a Grant-in-Aid for Scientific

Research (C) from the Ministry of Education, Culture, Sports, Science and Technology of Japan

Keywords:

Multiple sclerosis

Memory B cell

Naïve B cell

Cluster of differentiation

Interferon 


\section{ABSTRACT}

Memory and naïve B cells are considered to play distinct roles in immune regulation. However, the roles of memory and naïve B-cell subsets in multiple sclerosis (MS) have not yet been elucidated. In this study, we examined whether memory and naïve B-cell subsets differ between patients with MS and healthy subjects and whether interferon beta (IFN $\beta$ )-1b can affect these subsets in patients with MS. We also studied these subsets in relapsing and remitting stages of MS. Subjects included 31 patients with relapsing-remitting MS in the remitting stage, of which 15 were treated with IFN $\beta-1 \mathrm{~b}$ and 16 were not treated, and 22 healthy control subjects. For 11 of the 16 untreated patients, blood samples were also obtained in the relapsing stage. Expression of CD5, CD80, CD86, CCR5, CXCR3, CD11a, and CD49d in memory and naïve B cells in blood samples was examined by flow cytometry. The percentages of $\mathrm{CD} 86^{+}$cells and $\mathrm{CCR} 5^{+}$cells in the naïve B-cell subset were significantly higher in untreated patients than in control subjects or IFN $\beta$-treated patients. In patients with MS, the percentages of $\mathrm{CD} 86^{+}$cells and $\mathrm{CCR} 5^{+}$cells in the naïve B-cell subset and the percentage of $\mathrm{CD}^{+}$cells in the memory B-cell subset were significantly greater in the remitting stage than in the relapsing stage. These results indicate that memory and naïve B-cell subsets, especially $\mathrm{CD} 86^{+}$naïve $\mathrm{B}$ cells, $\mathrm{CCR} 5^{+}$naïve $\mathrm{B}$ cells, and $\mathrm{CD} 5^{+}$memory $\mathrm{B}$ cells, might be useful in the study of the pathogenesis of and therapy for MS. 
Multiple sclerosis (MS) is a major inflammatory demyelinating disease of the central nervous system (CNS) and it has been considered that MS is a T-cell-mediated autoimmune disease, involving inflammatory demyelination of the CNS by $\mathrm{CD}^{+}{ }^{+} \mathrm{T}$ cells specific for myelin oligodendrocyte glycoprotein and other CNS autoantigens [22]. However, one of the characteristics of MS is the persistent intrathecal synthesis of immunoglobulins, particularly oligoclonal $\mathrm{IgG}$, and reassessment of the role of B cells in the pathogenesis of MS is ongoing. In recent years, B cells have emerged as a novel therapeutic target for treating MS, and clinical trial data with rituximab, a B-cell-depleting antibody, provides reciprocal conceptual support for a prominent role of B cells in the pathogenesis of MS [9]. B cells are increasingly recognized as major players in the pathogenesis of MS [5]; however, not all subsets of B cells are considered important. Among T cells, for example, some subsets, such as Th17 cells, are important in the pathogenesis of MS; however, regulatory T cells can ameliorate symptoms of MS [for a review see 2]. Among B cells, the same scenario may be considered. For example, naïve and memory B-cell subsets have distinct, active roles in the regulation of normal immune responses, and abnormalities in these functions may contribute to human autoimmune disease [6]. Memory B cells are significantly distinct from naïve B cells, and the production of different effector cytokine profiles is a fundamental characteristic distinguishing naïve $\left(\mathrm{CD} 27^{-}\right)$from memory $\left(\mathrm{CD} 27^{+}\right)$human B cells [6]. However, it is not clear whether subsets of memory and naïve B cells differ in patients with MS and healthy individuals or whether these subsets are altered in relapsing and remitting stages of MS.

Interferon beta (IFN $\beta$ ) is widely used in the treatment of MS. Laboratory and clinical studies suggest that IFN $\beta$ acts on various processes in the immune system to modify cytokine production, inhibit T-cell activation, and decrease the expression of major histocompatibility complex class II molecules [14]. Major targets of studies on the function of IFN $\beta$ include T cells and antigen-presenting cells; the effect of IFN $\beta$ on B cells has not yet been studied 
extensively. Thus, it is not clearly understood whether memory and naïve B-cell subsets are affected by IFN $\beta$.

In this study, we focused on seven subsets of memory and naïve B cells in MS: CD5, CD80, CD86, CXCR3, CCR5, CD11a, and CD49d. CD5-positive B cells produce low-affinity polyreactive autoantibodies that recognize a variety of CNS antigens [16], and there are a few studies reporting that CD5 expression on B lymphocytes is higher in patients with active MS [3,18]. CD80 and CD86 are major costimulatory signals for T-cell activation, and variation in the expression of these proteins is likely to influence immune regulation in MS [11]. CXCR3 and CCR5 are chemokine receptors. The expression of CXCR3 in B cells in the cerebrospinal fluid in active MS has been reported to be significantly higher than that in control subjects [19], and patients with active MS show higher B-cell expression of CCR5 in the blood [19]. CD11a and CD49d are adhesion molecules, and it has been shown that B cells, in particular memory B cells, express a high level of very late antigen-4 (VLA-4; CD49d/CD29) $[1,15]$. We examined whether memory and naïve B-cell subsets differ between patients with MS and healthy control subjects and whether IFN $\beta-1 \mathrm{~b}$ can affect these subsets in patients with MS. We also studied alterations in these subsets in relapsing and remitting stages of MS.

Patients with clinically confirmed relapsing-remitting MS diagnosed according to the Poser criteria [17] were recruited at Hokkaido University Hospital, and 22 healthy control subjects were recruited from university staff personnel (female/male $=18 / 4$, mean age $=34.0$ years). All of the patients in this study had "conventional" MS as described previously [7]. Fifteen patients treated with IFN $\beta-1 \mathrm{~b}($ female $/$ male $=12 / 3$, mean age at blood sampling $=$ 36.5 years, mean age at disease onset $=28.3$ years, mean EDSS $=2.7)$ and 16 patients not treated with any disease-modifying therapy, including IFN $\beta-1 \mathrm{~b}($ female $/$ male $=13 / 3$, mean age at blood sampling $=36.4$ years, mean age at disease onset $=27.9$ years, mean EDSS $=$ 
2.7) were included. None of the patients had received any immunosuppressants, and none were exposed to steroids for at least 2 months before blood sampling. In the IFN $\beta$-1b-treated group, patients received IFN $\beta-1$ b subcutaneously every other day for at least 6 months. In the untreated group, patients had never received IFN $\beta-1 \mathrm{~b}$ or had not received it for at least 1 year. Of the 16 untreated patients, 11 experienced relapse, and blood samples were collected before the administration of steroid therapy (mean EDSS at relapse and remitting $=3.0$ and 1.8, respectively). Subject recruitment followed institutional review board approval, and informed consent was obtained from all subjects. All participants in this study were Japanese residents of Hokkaido, the northernmost island of Japan. Differences in sex ratio and age between patients and control subjects were not significant $(P>0.05)$.

The following directly conjugated antibodies were used: anti-CD5 phycoerythrin (PE), anti-CD11a PE, anti-CD19 phycoerythrin-cyanin 5 (PE-Cy5), anti-CD27 fluorescein isothiocyanate (FITC), anti-CD49d PE, anti-CD80 PE, anti-CD86 PE (all from BD Biosciences, San Jose, CA), anti-CXCR3 PE, and anti-CCR5 PE (R\&D Systems, Minneapolis, MN). B cells were detected with anti-CD19 antibody. CD27 was used as a marker of memory B cells; CD27-negative B cells were considered to be naïve B cells. Isotype controls included FITC-, PE-, and PE-Cy5 conjugated mouse IgG1 (BD Biosciences). Whole blood staining was conducted using the standard BD protocol, and cells were analyzed without delay on a three-color FACScan flow cytometer with CellQuest software (Becton Dickinson) (Fig. 1).

All data are expressed as mean \pm standard deviation (SD). Associations among healthy control subjects (HC), patients with MS treated with IFN $\beta-1 b$ (IFN-MS), and untreated patients with MS (non-IFN-MS) were tested by analysis of variance (ANOVA) followed by Tukey multicomparison test (GraphPad software; GraphPad Software, Inc., La Jolla, CA). Wilcoxon signed-rank test was used for comparison of B cells in relapsing and 
remitting stages. A 5\% significance level was used.

First, we compared the expression of CD27, CD5, CD80, CD86, CD11a, CD49d, CXCR3, and CCR5 in B cells among the HC, IFN-MS, and non-IFN-MS groups (Fig. 2). The percentages of $\mathrm{CD} 27^{+} \mathrm{B}$ lymphocytes in the IFN-MS group were significantly less than those in the control and non-IFN-MS groups $(P<0.001$ and $P<0.01$, respectively). The percentages of $\mathrm{CD} 80^{+}$B lymphocytes in the IFN-MS group were also significantly less than those in the control and non-IFN-MS groups $(P<0.01$ and $P<0.05$, respectively). However, the IFN-MS group showed lower CD86 positivity than the non-IFN-MS group $(P<0.01)$. There were no significant differences in mean fluorescence intensity (MFI) of CD11a and CD49d among the three groups in B cells. With respect to chemokine receptors, CCR5 positivity in B cells in the non-IFN-MS group was significantly higher than in the HC and IFN-MS groups $(P<0.05$ and $P<0.01$, respectively).

The percentages of $\mathrm{CD}^{+}$cells and $\mathrm{CD} 0^{+}$cells in memory and naïve B cells did not differ among the non-IFN-MS, HC, and IFN-MS groups (Fig. 3). CD86 positivity in memory B cells in the HC group was significantly less than that in the non-IFN-MS and IFN-MS groups ( $P<0.05$ and $P<0.001$, respectively). However, the percentage of CD86-positive cells among naïve B cells in the non-IFN-MS group was significantly higher than those in the HC and IFN-MS groups (both $P<0.05$ ). Among memory B cells, the IFN-MS group showed lower CXCR3 positivity than the HC group $(P<0.05)$, and there were no significant differences in CXCR3 among the three groups in naïve B cells. The percentage of CCR $5^{+}$ cells among naïve B cells in the non-IFN-MS group was significantly higher than that in the HC and IFN-MS groups $(P<0.05$ and $P<0.01$, respectively). With respect to adhesion molecules, there were no significant differences in MFI for CD11a or CD49d among the three groups in memory and naïve B cells.

We then compared data between relapsing and remitting MS (Fig. 4). There were no 
significant differences in the percentages of $\mathrm{CD}^{+}$cells and $\mathrm{CXCR}^{+}$cells or in the MFI of CD11a or CD49d in B cells between remitting and relapsing stages. With respect to CD27, CD80, CD86, and CCR5, significant increases in positive B cells were identified in the remitting stage $(P<0.05, P<0.01, P<0.05$, and $P<0.01$, respectively).

We also compared surface markers on memory and naïve B cells in relapsing and remitting stages (Fig. 5). The percentage of $\mathrm{CD}^{+}$cells among memory B cells was decreased in the relapsing stage compared to the remitting stage $(P<0.05)$. The percentage of $\mathrm{CD} 80^{+}$ cells in both memory and naïve B cells was decreased in the relapsing stage compared to the remitting stage $\left(P<0.05\right.$ and $P<0.01$, respectively). The percentage of $C D 86^{+}$cells among naïve B cells was decreased in the relapsing stage compared to the remitting stage $(P<0.05)$. The percentage of $\mathrm{CCR} 5^{+}$cells among both memory and naïve B cells was decreased in the relapsing stage compared to the remitting stage (both $P<0.01$ ).

Not only are memory B cells more easily activated to differentiate to plasma cells than naïve B cells, they also produce more proinflammatory cytokines, such as tumor necrosis factor (TNF) $\alpha$, interleukin (IL)-6, and lymphotoxin, which could further amplify the ongoing immune response [6]. TNF $\alpha$ itself has been shown to be toxic to oligodendrocytes [10], suggesting roles for effector B-cell cytokines that may contribute to disease independent of antibody secretion [5]. However, naïve B cells produce more the downregulatory cytokine IL-10 than do memory B cells [6]. Taken together, these results suggest that naïve and memory B-cell subsets play distinct, active roles in the regulation of normal immune responses and indicate that abnormalities in these functions may contribute to MS [6]. In the present study, we demonstrated that detailed subsets of memory and naïve B cells differ between patients with MS and healthy control subjects, and these differences may suggest targets for MS therapies. The major results of the present study are that patients with MS have significantly higher rates of positivity for CD86 and CCR5 in naïve B cells compared to 
healthy individuals. In addition, in patients treated with IFN $\beta-1 b$, the percentages of CD $86^{+}$ and $\mathrm{CCR} 5^{+}$naïve B cells were significantly decreased compared to those in untreated patients, although there were no significant differences in CD86- or CCR5-positivity in memory B cells between IFN $\beta$-treated and untreated patients. Thus, a decrease in $\mathrm{CD}^{+} 6^{+}$and $\mathrm{CCR}^{+}$cells among naïve B cells may be associated with the critical effects of IFN $\beta$ in MS. IFN $\beta$ is the most widely prescribed treatment for relapsing-remitting MS, reducing exacerbations and disease progression by approximately $35 \%$ [20]. There have been many studies on the mode of action of IFN $\beta$ in MS, including immune and cell cycle regulation, modulation of cytokines, and expression of cell-surface molecules. However, there has not been extensive research on the effects of IFN $\beta$ on B cells. Recently, it was reported that IFN $\beta$ therapy induces the expression of a potent B-cell survival factor, B-cell activating factor of the TNF family (BAFF) [12], although the function of this family in MS has not yet been demonstrated clearly. Therapy with IFN $\beta-1 b$ markedly reduces the number of circulating $\mathrm{CD}^{+} 0^{+} \mathrm{B}$ cells $[8]$, and our present results also demonstrated that the rates of $\mathrm{CD} 80^{+} \mathrm{B}$ cells were decreased in IFN $\beta$-treated patients compared to untreated patients. There were no significant differences in CD80 positivity in memory and naïve B cells between IFN $\beta$-treated and untreated patients, although in naïve B cells, there was a tendency toward a decrease in CD80 positivity in IFN $\beta$-treated patients compared to untreated patients.

CD86 as well as CD80 on B cells bind to CD28 or cytotoxic T-lymphocyte antigen (CTLA)-4 on T cells, which leads to either costimulation or coinhibition, depending on the precise expression patterns of receptors and ligands and the state of activation of the two cell types [4]. CD28 is expressed on resting T cells after engagement by CD86, leading to activation of resting $\mathrm{T}$ cells. This costimulation leads to increased production of growth factors, such as IL-2, and increased cell-survival signals in T cells [4]. However, engagement 
of CTLA-4 by CD86 results in the attenuation of T-cell activation [4]. The complex functions of CD86 may be associated with our results; our data suggest that greater CD86 positivity in naïve B cells in untreated patients might be associated with T-cell activation, and the decrease in CD86 expression in naïve B cells during relapse might be associated with attenuation of T-cell activation.

The expression of peripheral blood CCR $5^{+} \mathrm{B}$ cells has been reported to be higher in MS patients compared to healthy control subjects $[13,19]$, and there is a trend toward a decrease in CCR5 positivity in response to IFN $\beta$ in relapsing-remitting MS [19]. Our present data are consistent with these findings. Furthermore, we found a decrease in CCR5 positivity in response to IFN $\beta$ only in naïve B cells. However, the expression of CCR5 in both memory and naïve B cells was increased in the remitting stage compared to the relapsing stage of MS. Recent studies with biopsy materials demonstrated that macrophages/microglia within early remyelinating lesions in MS express predominantly CCR5, suggesting a possible role of $\mathrm{CCR}^{+}$cells in initiating remyelination [21]. The increase in $\mathrm{CCR}^{+} \mathrm{B}$ cells might be associated with such a role.

There could be distinct and proactive roles of memory and naïve human B-cell subsets in the regulation of memory immune responses and in autoimmunity [6]. Our present data suggest that more detailed research on subsets of memory and naïve B cells, especially $\mathrm{CD}^{+} 6^{+}$naïve $\mathrm{B}$ cells, $\mathrm{CCR}^{+}$naïve $\mathrm{B}$ cells, and $\mathrm{CD}^{+}$memory $\mathrm{B}$ cells, in MS will be important. Further studies on the functions of memory and naïve B-cell subsets may shed light on B-cell interactions in MS and provide a novel therapeutic approach for the treatment of MS. 


\section{References}

[1] A. Alter, M. Duddy, S. Hebert, K. Biernacki, A. Prat, J.P. Antel, V.W. Yong, R.K. Nuttall, C.J. Pennington, D.R. Edwards, A. Bar-Or, Determinants of human B cell migration across brain endothelial cells, J. Immunol.170 (2003) 4497-4505.

[2] A. Awasthi, G. Murugaiyan, V.K. Kuchroo, Interplay between effector Th17 and regulatory T cells, J. Clin. Immunol. 28 (2008) 660-670.

[3] P. Bongioanni, C. Fioretti, R. Vanacore, F. Bianchi, F. Lombardo, F. Ambrogi, G. Meucci, Lymphocyte subsets in multiple sclerosis. A study with two-colour fluorescence analysis, J. Neurol. Sci. 139 (1996) 71-77.

[4] M. Collins, V. Ling, B.M. Carreno, Immortalized human microglial cell line: phenotypic expression, Genome Biol. 6 (2005) 223.

[5] M. Duddy, A. Bar-Or, B-cells in multiple sclerosis, Int. MS. J. 13 (2006) 84-90.

[6] M. Duddy, M. Niino, F. Adatia, S. Hebert, M. Freedman, H. Atkins, H.J. Kim, A. Bar-Or, Distinct effector cytokine profiles of memory and naive human B cell subsets and implication in multiple sclerosis, J. Immunol. 178 (2007) 6092-6099.

[7] T. Fukazawa, K. Miyasaka, K. Tashiro, T. Hamada, F. Moriwaka, T. Yanagihara, K. Hamada, MRI findings of multiple sclerosis with acute transverse myelopathy, J. Neurol. Sci. 110 (1992) 27-31.

[8] K. Genç, D.L. Dona, A.T. Reder, Increased CD80+ B cells in active multiple sclerosis and reversal by interferon $\beta-1 b$ therapy, J. Clin. Invest. 99 (1997) 2664-2671.

[9] S.L. Hauser, E. Waubant, D.L. Arnold, T. Vollmer, J. Antel, R.J. Fox, A. Bar-Or, M. Panzara, N. Sarkar, S. Agarwal, A. Langer-Gould, C.H. Smith; HERMES Trial Group, B-cell depletion with rituximab in relapsing-remitting multiple sclerosis, N. Engl. J. Med. 358 (2008) 676-688.

[10] A. Jurewicz, M. Matysiak, K. Tybor, K. Selmaj, TNF-induced death of adult human 
oligodendrocytes is mediated by c-jun NH2-terminal kinase-3, Brain. 126 (2003) $1358-1370$.

[11] V.K. Kuchroo, M.P. Das, J.A. Brown, A.M. Ranger, S.S. Zamvil, R.A. Sobel, H.L. Weiner, N. Nabavi, L.H. Glimcher, B7-1 and B7-2 costimulatory molecules activate differentially the Th1/Th2 developmental pathways: application to autoimmune disease therapy, Cell. 80 (1995) 707-718.

[12] M. Krumbholz, H. Faber, F. Steinmeyer, L.A. Hoffmann, T. Kümpfel, H. Pellkofer, T. Derfuss, C. Ionescu, M. Starck, C. Hafner, R. Hohlfeld, E. Meinl, Interferon-beta increases BAFF levels in multiple sclerosis: implications for B cell autoimmunity, Brain. 131 (2008) 1455-1463.

[13] E.M. Martínez-Cáceres, C. Espejo, L. Brieva, I. Pericot, M. Tintoré, I. Sáez-Torres, X. Montalban, Expression of chemokine receptors in the different clinical forms of multiple sclerosis, Mult. Scler. 8 (2002) 390-395.

[14] O. Neuhaus, B.C. Kieseier, H.P. Hartung, Pharmacokinetics and pharmacodynamics of the interferon-betas, glatiramer acetate, and mitoxantrone in multiple sclerosis, J. Neurol. Sci. 259 (2007) 27-37.

[15] M. Niino, C. Bodner, M.L. Simard, S. Alatab, D. Gano, H.J Kim, M. Trigueiro, D. Racicot, C. Guérette, J.P. Antel, A. Fournier, F. Grand'Maison, A. Bar-Or, Natalizumab effects on immune cell responses in multiple sclerosis, Ann. Neurol. 59 (2006) 748-754.

[16] J.O. Pers, C. Jamin, F. Predine-Hug, P. Lydyard, P. Youinou, The role of CD5-expressing B cells in health and disease, Int. J. Mol. Med. 3 (1999) 239-245.

[17] C.M. Poser, D.W. Paty, L. Scheinberg, W.I. McDonald, F.A. Davis, G.C. Ebers, K.P. Johnson, W.A. Sibley, D.H. Silberberg, W.W. Tourtellotte, New diagnostic criteria for multiple sclerosis: guidelines for research protocols, Ann. Neurol. 13 (1983) 227-231.

[18] T.F. Scott, J. McKolanis, W. Rothfus, E. Cottington, Lymphocyte subsets in relapsing-remitting multiple sclerosis: a longitudinal study of B lymphocytes and $\mathrm{T}$ 
lymphocytes, Neurol. Res. 16 (1994) 385-388.

[19]T.L. Sørensen, H. Roed, F. Sellebjerg, Chemokine receptor expression on B cells and effect of interferon- $\beta$ in multiple sclerosis, J. Neuroimmunol.122 (2002) 125-131.

[20] A. Tourbah, O. Lyon-Caen, Interferons in multiple sclerosis: ten years' experience, Biochimie. 89 (2007) 899-902.

[21] C. Trebst, F. König, R. Ransohoff, W. Brück, M. Stangel, CCR5 expression on macrophages/microglia is associated with early remyelination in multiple sclerosis lesions, Mult. Scler. 14 (2008) 728-733.

[22] K.C. Williams, E. Ulvestad, W.F. Hickey, Immunology of multiple sclerosis, Clin. Neurosci. 2 (1994) 229-245. 


\section{Figure Legends}

Fig. 1. Sample scatter plots are shown. CD19 $9^{+}$B cells were quantified by CD 27 and each of CD5, CD80, CD86, CXCR3, CCR5, CD11a, and CD49d.

Fig. 2. Comparison of CD27-, CD5-, CD80-, CD86-, CXCR3-, and CCR5-positive cells in B cells and MFI of CD49d and CD11a in B cells among healthy controls $(\mathrm{HC} ; \mathrm{n}=22)$, patients with MS treated with IFN $\beta-1 b($ IFN-MS; $\mathrm{n}=15)$, and untreated patients (non-IFN-MS; $\mathrm{n}=$ 16). ${ }^{*} P<0.05,{ }^{* *} P<0.01,{ }^{* * *} P<0.001$ by ANOVA followed by Tukey multicomparison test.

Fig. 3. Comparison of CD5-, CD80-, CD86-, CXCR3-, and CCR5-positive cells in memory and naïve B cells and MFI of CD11a and CD49d in memory and naïve B cells among healthy controls $(\mathrm{HC} ; \mathrm{n}=22)$, patients with MS treated with IFN $\beta-1 \mathrm{~b}($ IFN-MS; $\mathrm{n}=15)$, and untreated patients (non-IFN-MS; $\mathrm{n}=16$ ). ${ }^{*} P<0.05, * * P<0.01, * * * P<0.001$ by ANOVA followed by Tukey multicomparison test.

Fig. 4. Comparison of CD27-, CD5-, CD80-, CD86-, CXCR3-, and CCR5-positive cells in B cells and MFI for CD11a and CD49d in B cells between relapsing and remitting stages of MS (n $=11) .{ }^{*} P<0.05, * * P<0.01,{ }^{* * *} P<0.001$ by Wilcoxon signed-rank test.

Fig. 5. Comparison of CD5-, CD80-, CD86-, CXCR3-, and CCR5-positive cells in memory and naïve B cells and MFI for CD11a and CD49d in memory and naïve B cells between relapsing and remitting stages of MS $(\mathrm{n}=11) .{ }^{*} P<0.05, * * P<0.01, * * * P<0.001$ by Wilcoxon signed-rank test. 
Fig. 1
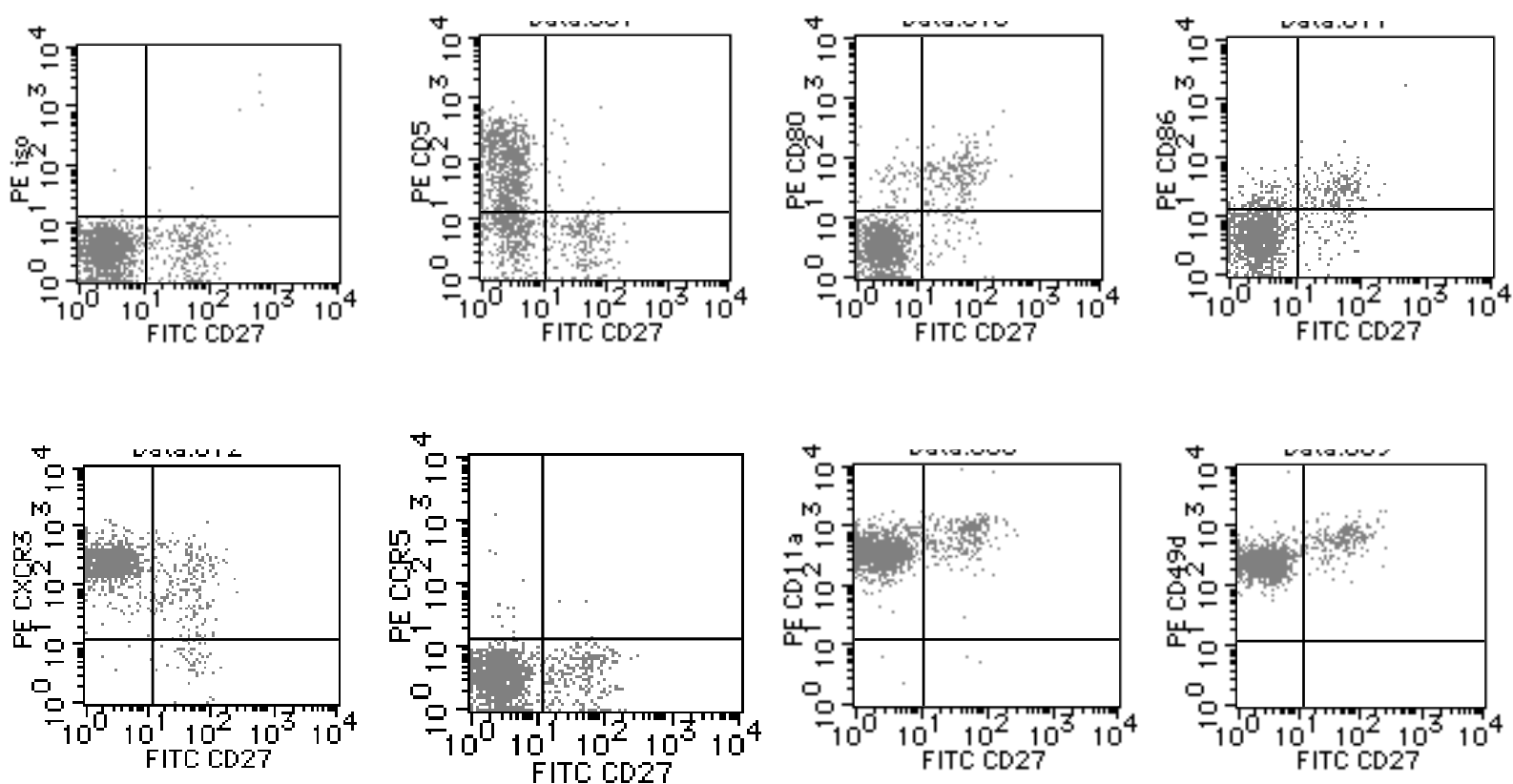
Fig. 2
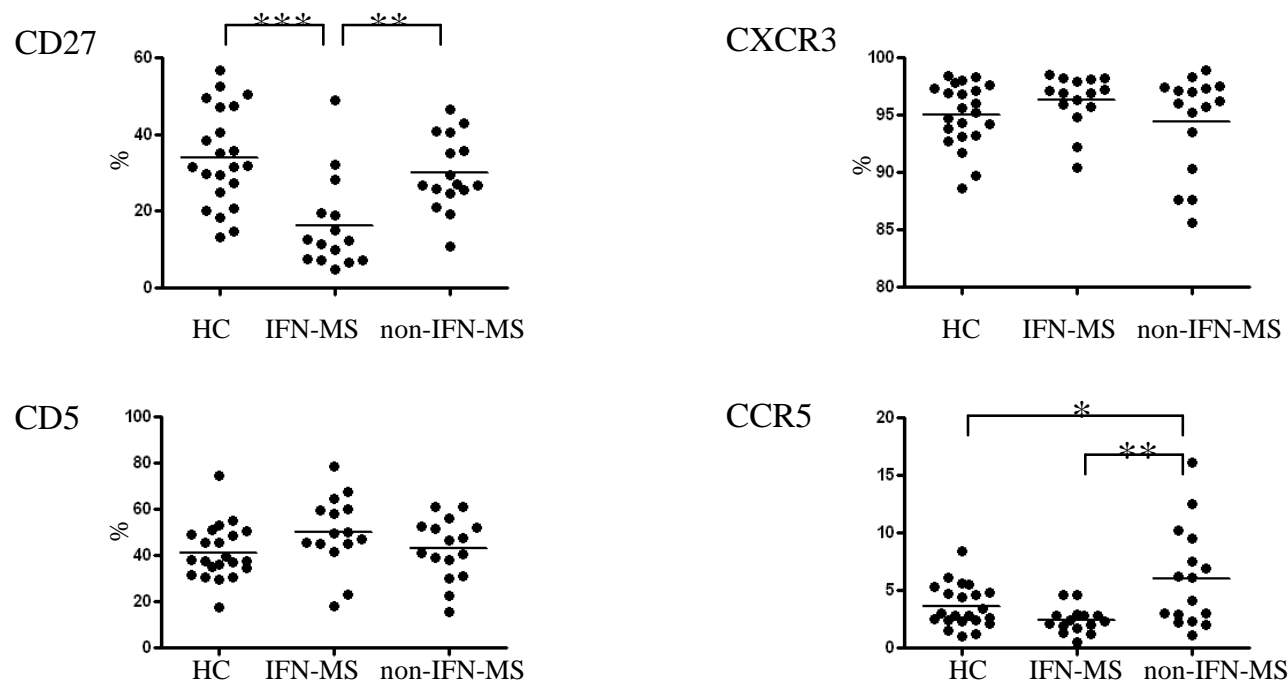

CCR5

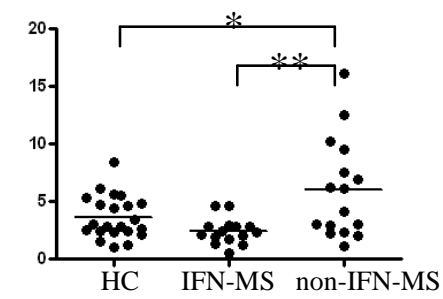

CD80

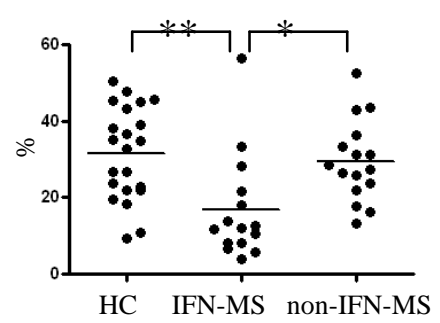

CD86

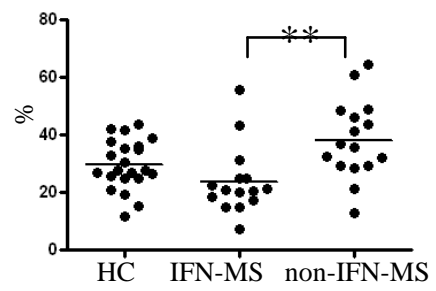

CD11a

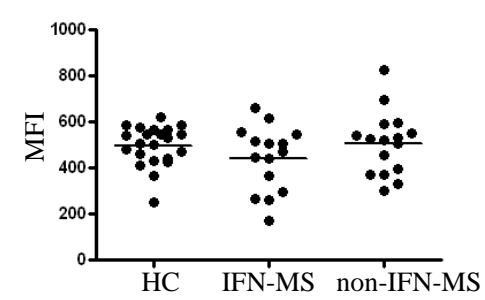

CD49d

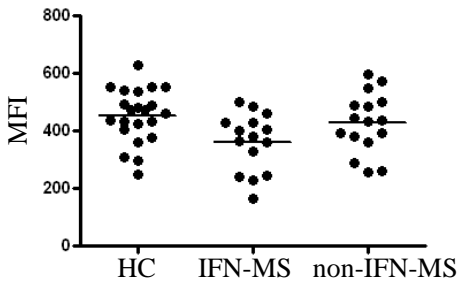


Fig. 3

$\mathrm{CD}^{2} 7^{+}$Memory B cells
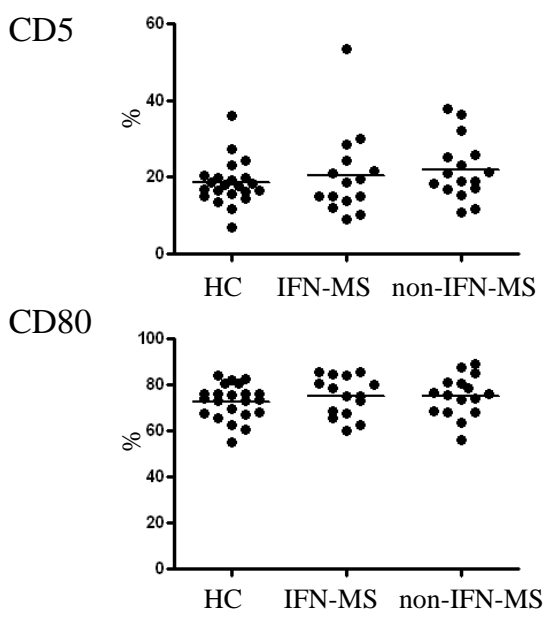

CD86

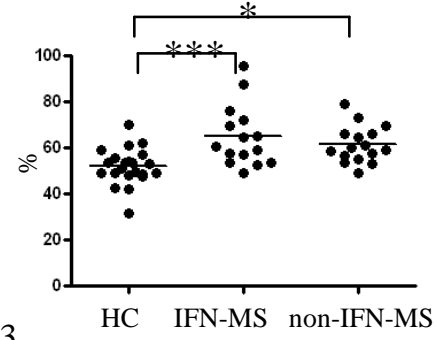

CXCR3

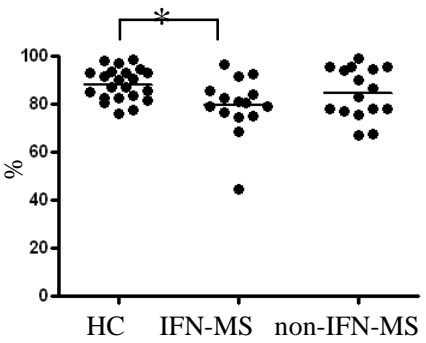

CCR5

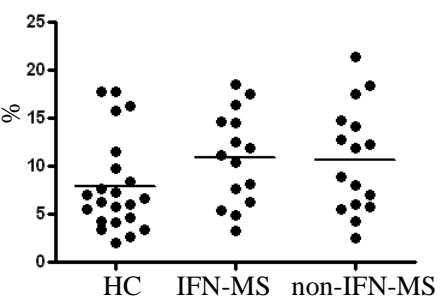

CD11a
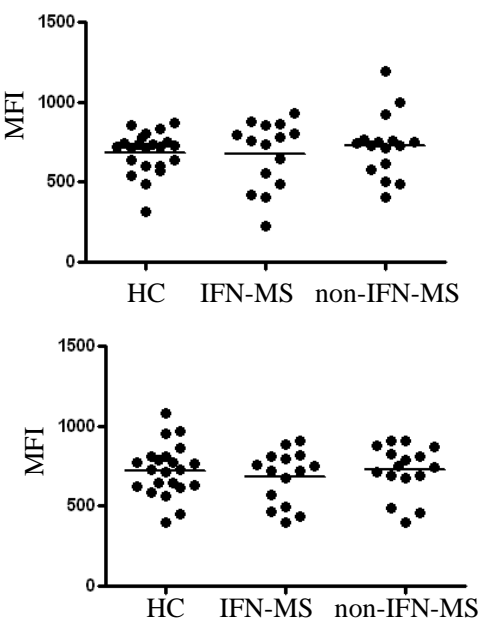

CD27- Naïve B cells
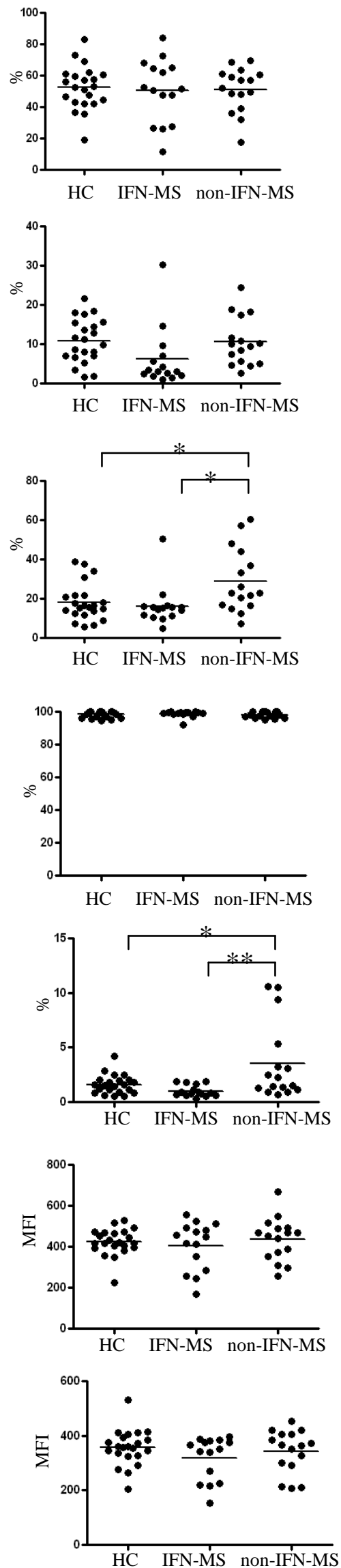
Fig. 4
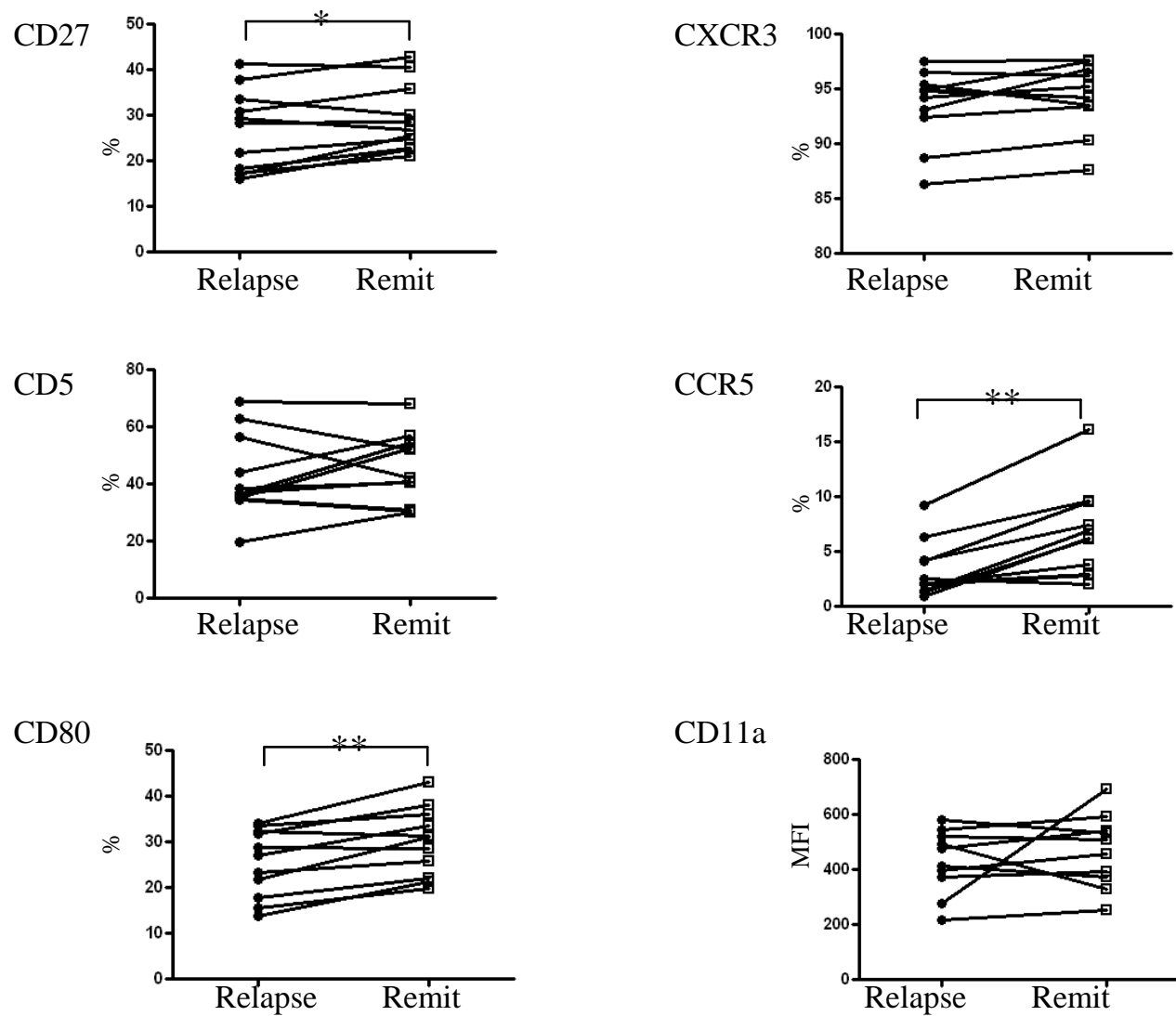

CD11a

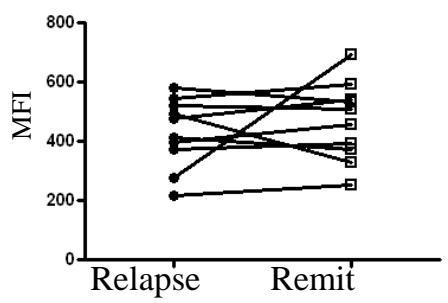

CD86

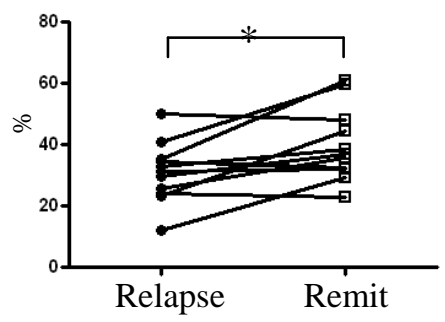

CD49d

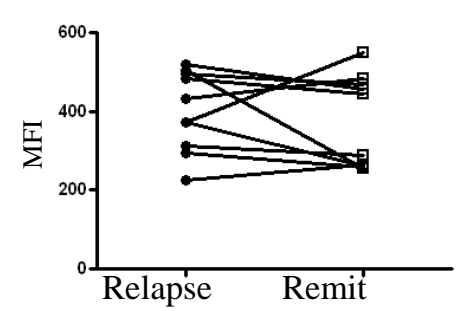


Fig. 5

CD5

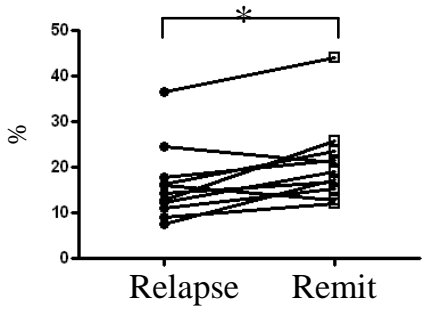

CD80

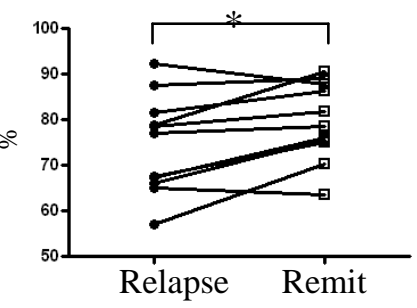

CD86

CXCR3
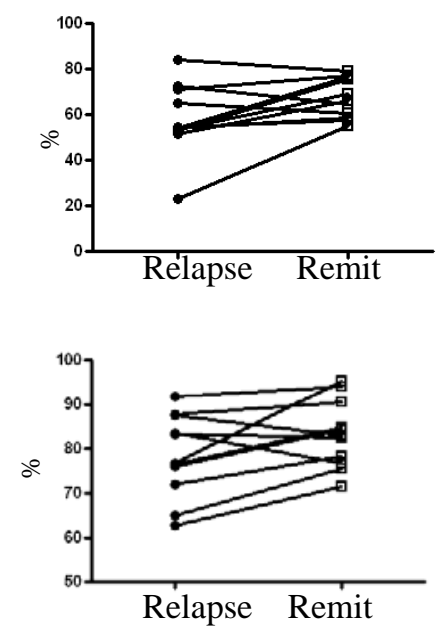

CCR5

CD11a

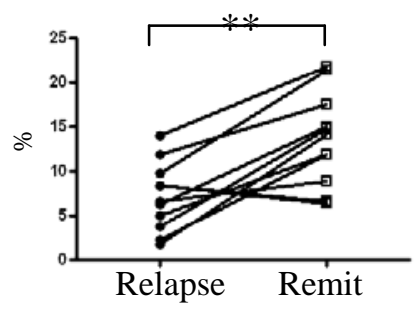

CD49d
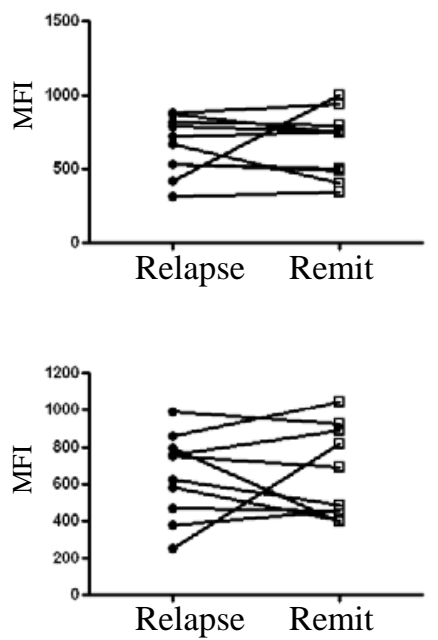

CD27- Naïve B cells
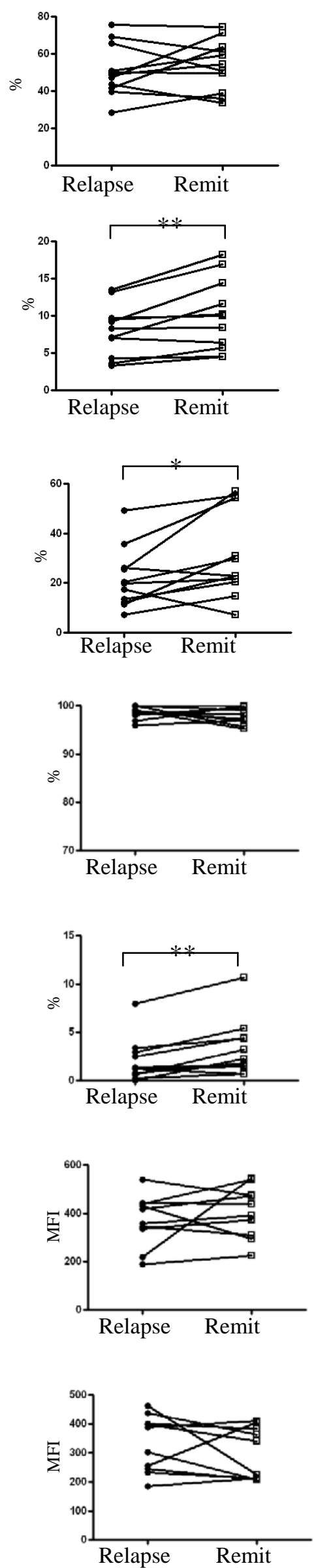